\title{
Incorporating Doppler Velocity Measurement for Track Initiation and Maintenance
}

\author{
F Kural, METU Technopolis \\ F Arikan, Hacattepe University \\ O Arikan, Bilkent University \\ M Efe, Ankara University \\ Turkey
}

() The IEE

Printed and published by the IEE, Michael Faraday House, Six Hills Way,

Stevenage, Herts SG1 2AY, UK 


\section{About the Speaker}

Dr. Efe got his PhD in 1998 from the University of Sussex at Brighton in the UK. He is currently an Assistant Professor at Electronics Engineering Department at Ankara University, Turkey. His research interests include target tracking, dynamic estimation and data fusion. 


\section{Incorporating Doppler Velocity Measurement for Track Initiation and Maintenance \\ Faruk Kural', Feza Arıkan ${ }^{2}$, Orhan Arıkan ${ }^{3}$, Murat Efe ${ }^{4}$ \\ 'Optimal Inc., METU Technopolis, Ankara, Turkey, kural_faruk@yahoo.com \\ ${ }^{2}$ Hacettepe University, Dept. Electrical and Electronics Eng., Ankara, Turkey, arikan@ee.hacettepe.edu.tr \\ ${ }^{3}$ Bilkent University, Dept. Electrical and Electronics Eng., Ankara, Turkey, oarikan@ee.bilkent.edu.tr \\ ${ }^{4}$ Ankara University, Electronics Eng. Dept., Ankara, Turkey, efe@eng.ankara.edu.tr}

Abstract Performance of multiple target tracking algorithms in complex environments heavily relies on the success of track initiation and measurement-to-track association algorithms. Doppler velocity measurement is the major discriminant of clutter from the target of interest with relatively higher velocities. This work summarizes the analytical derivations and presents simulation results about track initiation and maintenance using Doppler velocity reports along with the 3D position measurements extracted by a phased array radar.

Introduction

For Phased Array Radars (PAR), the performance of multiple target tracking algorithms in complex environments such as missing detections and false alarms caused by clutter heavily relies on the success of track initiation (TI) and measurement-to-track association approaches. In a tracking radar system, extracted measurements from the detections are transferred to the target tracking module with a transfer rate allowed by the PAR. The measurements are first fed into the measurement-to-track association unit. This unit correlates the measurements with the previously initiated tracks. The measurements which are not correlated with the registered tracks are assumed to be coming from new potential targets and they are directed to the TI unit. The performance of TI is essential to the tracking system. When a Tl unit fails to initiate real tracks, the radar may miss the opportunity of tracking and identification of potential targets. In the cases where $\mathrm{TI}$ initiates false tracks, limited energy of the radar is wasted on the computational complexity of the following track update algorithms, resulting in the reduction of the number of targets to be tracked. Thus, it is very critical for the TI unit to correctly initiate the real tracks in a required period of time. Furthermore, TI should also suppress the measurements originated from clutter. A statistically successful TI should be able to set its True Track Initiation Probability (TTIP) to an acceptable level while keeping the False Track Initiation Probability (FTIP) as low as possible. The studies which contain analytical expressions of $\mathrm{TI}$ algorithms such as the rule-based and the logicbased are very rare and limited $[1,2,3]$. Such studies are performed only for two dimensional position measurements, namely range and azimuth. However, the major discriminant of clutter from the target of interest with relatively higher velocities is the Doppler velocity measurements [4]. Although PARs are capable of providing additional return information such as elevation and Doppler velocity, there have not been any studies in the open literature where the Doppler measurements extracted by a PAR are included in the analytical derivations of $T$ I algorithms.
Besides, PARs are also capable of using an adaptive sampling policy by the agile beam positioning. Especially for multiple target environments with clutter, increasing the track update interval significantly reduces the signal processing computational complexity leading to conservation of radar energy for more demanding tasks such as simultaneous tracking of an increased number of targets $[5,6]$. However, longer track update intervals can also result in an increase in the state estimation errors which will reduce the probability of target detection. Thus, a major design criterion in PARs is increasing the track update interval while keeping the other performance criteria at preset levels. Interacting Multiple Model (IMM) Probabilistic Data Association Filter (PDAF) is the state of the art tracking algorithm that can be used successfully under such tradeoffs [5, 6]. Although the major discriminant of clutter from the desired target with relatively higher velocities is the Doppler velocity measurements, there has not been any studies in the open literature where the Doppler measurements extracted by a PAR are included in IMMPDAF with adaptive sampling to track highly manoeuvring targets in complex environments.

In this study, the elevation and Doppler velocity measurements are incorporated into the commonly used $\mathrm{TI}$ algorithms for the first time. With this study, the measurement set is expanded from a merely range and azimuth to include elevation and Doppler velocity. The direction of the Doppler velocity in the consecutive measurements is also checked to better identify the correct tracks. Besides, Doppler velocity is incorporated into IMMPDAF with adaptive sampling policy and the performance is compared with the position only measurements case for various benchmark targets.

Track Initation Algorithms with Position Only Measurements

In radar tracking systems, there exist two widely used $\mathrm{TI}$ algorithms: the rule-based Tl [1] and the logicbased TI $[1,2,3]$. The rule-based TI method uses two basic rules to initiate tracks:

1. The estimated velocity based on position reports given in Cartesian coordinates must be inside the 
preset minimum and maximum velocity thresholds $\left(0 \leq v_{\text {max }}<v_{\text {mise }}\right)$. For $M$ sampling interval $\mathrm{TI}$, such a velocity gating is expressed as [1]:

$$
v_{\text {min }}<\left\|\mathbf{r}_{j i+1}(1: 3)-\mathbf{r}_{k i, i}(1: 3)\right\| / t_{0}<v_{\text {mat }}
$$

where $\quad \mathbf{r}_{i, i}=\left[\begin{array}{llll}x_{k, i} & y_{k, i} & z_{k, i} & v_{k, 1}\end{array}\right]^{\mathrm{T}}$ is the $k^{\text {th }}$ measurement vector including $3 \mathrm{D}$ position and Doppler velocity at the $i^{\text {th }}(i=1,2, \ldots, M-1)$ sampling (transfer) interval, $t_{s}$ is the sampling interval of all the reports inside the search volume to Tl unit and $\|$.$\| is the L_{2}$ vector norm operator.

2. The estimated acceleration must be below the maximum allowed acceleration threshold level, $a_{\text {mar }}$ :

$$
\left.\|\left(\mathbf{r}_{t, i+1}(1: 3)-\mathbf{r}_{t, i}(1: 3)\right) / t_{s}^{2}-\left(\mathbf{r}_{t, i}(1: 3)-\mathbf{r}_{t, i-1}(1: 3)\right)\right) / t_{a}^{2} \|<\boldsymbol{a}_{\max }
$$

The logic-based $\mathrm{TI}$ methos is as follows $[1,2,3]$.

1. For the first two sampling times, the logic-based $\mathrm{TI}$ method is the same as the rule-based one. In this method, a velocity vector is estimated using the measurements one from each sampling time by

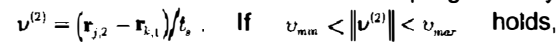
potential tracks are formed and position predictions are made for the third sampling time as $\mathbf{r}^{(3)}=\mathbf{r}_{j, 2}+\nu^{(2)} t_{\mathrm{f}}$.

2. At the third sampling time, a validation gate of radius $r_{0}$ is set up. Any measurement that falls inside the gate $\left\|r_{k, j}-r^{(3)}\right\| \leq r_{0}$ will extend the potential track. Using the report inside the gate, an acceleration estimation, $a^{(3)}=\left(\nu^{(3)}-\nu^{(2)}\right) / t$, , and a

position prediction, $\mathbf{r}^{(4)}=\mathbf{r}_{k, 3}+\boldsymbol{v}^{(3)} t_{\mathrm{s}}+0.5 \mathbf{a}^{(3)} t_{\mathrm{s}}^{2}$, for the next sampling time are made. If more than one measurement satisfies the gating requirements, the report which has the nearest distance to the predicted position is chosen. In case of no measurement inside the gate, potential track is deleted.

3. The same procedures are repeated for $M$ sampling times. Every potential track satisfying all the requirements is declared to be initiated.

FTIP Analyses of TI Algorithms with Position Only Measurements

Firstly, FTIP analysis of the rule-based $\mathrm{II}$ is assumed. Clutter based reports degrade the performance of tracking systems by increasing FTIP. Therefore, FTIP is a critical parameter of TI systems. Herein, clutter based reports in Cartesian coordinates $\left(x_{i}, y_{i}, z_{i}\right)$ are assumed to be uniformly distributed within the search volume of the PAR, $V$. The FTIPs at each sampling time, $P_{i}$, are also assumed to be independent of each other. Hence, the overall FTIP is $P_{r T I}=P_{1} P_{2} \ldots P_{M}$. Let the probability that any clutter based measurement falling inside the velocity gate given in Eq.(1) at the second sampling time be $p_{2}$. Taking into account the total number of resolution cells, $\zeta$, all with the same probability of false alarm, $P_{F A}$, in the search volume, the probability that at least one clutter based measurement falls into the region given by Eq.(1) is calculated as [3]:

$$
P_{2}=1-\exp \left(-P_{\boldsymbol{r}^{\prime}} \zeta \boldsymbol{p}_{2}\right)
$$

Recalling that the spatial distribution of clutter reports in the search volume is uniformand using the classical definition of probability, $p$. can be written as $p_{2}=V_{2} / V$. Here, $V_{2}$ is the volume of the velocity gate given in Eq.(1). Considering that a target with the minimum velocity $v_{m i n}$ and the maximum velocity $v_{\max }$, may move to any direction with the same probability between two sampling times, it is expected to be inside the spherical volumes with the radiuses of $R_{1}=v_{\min } t_{s}$ and $R_{2}=v_{\max } t_{s}$. Therefore, the occuring volume is calculated as $V_{2}=$ $(4 \pi / 3)\left(R_{2}^{3}-R_{1}^{3}\right)$. At the next sampling time, the measurements are to be subjected to both velocity and acceleration gating. For a given clutter report $\left(x_{2}, y_{2}, z_{2}\right)$ satisfying Eq.(1), velocity and acceleration gating necessitate $\left(x_{3}, \boldsymbol{y}_{3}, z_{3}\right)$ to satisfy the constraint $R_{3} \leq d_{2,3} \leq R_{4}$ where $R_{3}=\max \left(R_{1}, d_{1,2}-R_{a}\right), \quad R_{4}=$ $\min \left(R_{2}, d_{1,2}+R_{0}\right)$ [3]. Here, $R_{0} \triangleq a_{\max } t_{s}^{2}$ and $d_{i, 1+1}=$ $\sqrt{\left(x_{i}-x_{i+1}\right)^{2}+\left(y_{r}-y_{i+1}\right)^{2}+\left(z_{i}-z_{i+1}\right)^{2}}$. Therefore, at the third sampling time, the probability that at least one clutter reports satisfy acceleration gating given by Eq.(2), $P_{3}$, is similarly calculated as:

$$
P_{3}=1-\exp \left(-P_{F i} \zeta p_{3}\right)
$$

where $p_{3}$ is defined as the probability of accepting a clutter report $\left(x_{i 3}, y_{3}, z_{3}\right)$ to extend the track given that $\left(x_{1}, y_{1}, z_{1}\right)$ and $\left(x_{2}, y_{2}, z_{2}\right)$ satisfying Eq.(1) and calculated as:

$$
\begin{aligned}
& p_{3}= \\
& \qquad \begin{array}{l}
\left(\frac{1}{6}\left(R_{1}+R_{a}\right)^{6}-\frac{1}{6} R_{5}^{6}+\frac{3}{5} R_{a}\left(\left(R_{1}+R_{a}\right)^{5}-R_{1}^{5}\right)+\right. \\
\left.\frac{3}{4} R_{a}^{2}\left(\left(R_{1}+R_{a}\right)^{4}-R_{1}^{4}\right)+\frac{1}{3}\left(\left(R_{a}^{3}-R_{1}^{3}\right)\left(R_{1}+R_{a}\right)^{3}-R_{1}^{3}\right)\right) \\
+\left(\frac{6}{5} R_{a}\left(R_{2}-R_{a}\right)^{5}-\left(R_{1}+R_{a}\right)^{5}+\frac{2}{3} R_{a}^{3}\left(R_{2}-R_{a}\right)^{3}-\left(R_{1}+R_{a}\right)^{3}\right) \\
+\left(\frac{-1}{6} R_{2}^{6}+\frac{1}{6}\left(R_{2}-R_{a}\right)^{6}+\frac{3}{5} R_{a}\left(R_{2}^{5}-\left(R_{2}-R_{a}\right)^{6}\right)-\right. \\
\left.\frac{3}{4} R_{a}^{2}\left(R_{2}^{4}-\left(R_{2}-R_{a}\right)^{4}\right)+\frac{1}{3}\left(R_{2}^{3}+R_{a}^{3}\right)\left(R_{2}^{3}-\left(R_{2}-R_{a}\right)^{3}\right)\right)
\end{array}
\end{aligned}
$$

where, $\quad k=4 \pi\left(\theta_{i_{2}}-\theta_{i_{1}}\right)\left(\sin \phi_{h_{2}}-\sin \phi_{i_{1}}\right) /\left(3 V V_{2}\right), \quad \theta_{i_{1}}$ and $\theta_{b_{2}}\left(\theta_{t_{2}}>\theta_{t_{1}}\right)$ is the azimuthal coverage angles, $\phi_{t_{1}}$ and $\phi_{t_{2}}\left(\phi_{t_{2}}>\phi_{t_{1}}\right)$ is the coverage angles in elevation. If the number of total sampling intervals is chosen as $M=4$ and assuming that the influences

\footnotetext{
$1^{1}$ In [3], $R$, was calculated erroneously as $R_{a}=0.5 a_{\text {nox }} t_{a}^{2}$.
} 
of clutter reports at the first sampling time on the velocity and acceleration limiting at the fourth sampling time are negligible, $P_{4} \approx P_{3}$ can be written. Therefore, the overall FTIP, $P_{k^{\prime} T \text { r }}$ is calculated as:

$$
P_{f T J}=\left(1-\exp \left(-P_{F A} \zeta p_{2}\right)\right)\left(1-\exp \left(-P_{F A} \zeta p_{3}\right)\right)^{2}
$$

As for the FTIP analysis of the logic-based TI, since the same methodology with the rule-based one is used for the first two sampling times, probability $p_{2}$ is $p_{2}=V_{2} / V$. For the next sampling times, due to the fixed radius of gates, the volume of the gate is calculated as $V_{3}=4 \pi r_{0}^{3} / 3$. Therefore, probability $p_{3}$ is determined as $p_{3}=V_{3} / V$. Similarly, if $M=4$, the overall FTIP is calculated as in Eq.(6).

Track Initiation Algorithms with Position and Doppler Velocity Measurements

Using Doppler velocity measurements along with the 3D position measurements extracted by a PAR brings extra tests given in Eq.( $(7)$ to decrease FTIP:

$$
\left\{\begin{array}{l}
v_{\operatorname{mat}} \leq\left|\mathbf{r}_{i, i}(4)\right| \leq v_{\max } \cap\left|\mathbf{r}_{t, i}(4)-\mathbf{r}_{t, i-1}(4)\right| / t_{s} \leq a_{\max } \\
\cap \mathbf{r}_{t, i}(4) \mathbf{r}_{t, i-1}(4) \geq-\xi_{u}
\end{array}\right\}(7)
$$

where $r_{t, t}$ and $r_{t, i-1}$ the vectors in which there exist a set of measurements succeeded in passing position only based velocity and acceleration tests at $i^{\text {th }}$ and $(i-1)^{\text {th }}$ sampling times respectively, where $i=1,2,3$ for velocity test and $i=2,3$ for acceleration test. In Eq.(7), additional Doppler velocity report based thresholdings are applied to the measurements succeeded in passing the position only based tests at the preceding sampling times. The first two tests are based on the absolute value of the Doppler velocity measurement and the final one uses the direction of it. Firstly, absolute value of Doppler velocity measurement is tested whether it falls into the velocity gate. Then, absolute value of the acceleration based on the difference of the Doppler velocity measurements obtained at the successive sampling times is thresholded with a maximum acceleration level. Finally, taking into account the assumption that a complex true target cannot move to the opposite direction in a short enough period of sampling time, minimum change of the velocity of benchmark targets [7] in a sampling period based thresholding, $\xi_{v}$, is applied. Herein, due to the possible negative values of Doppler velocity reports, negative sign is used. Therefore, using these additional tests, clutter measurements which have relatively lower Doppler velocities than a complex true target are aimed to be removed.

FTIP Analysis of TI Algorithms with Position and Doppler Velocity Measurements

In this section, the newly obtained analytical expressions of FTIP with Doppler velocity measurements are given. In case of the existence of Doppler velocity measurements, at the $i^{\text {th }}$ sampling time, the probability of falling an undesired measurement inside the velocity gate for $i=1,2,3$ and acceleration gate for $i=2,3, p_{i}^{D}$ is defined as:

$$
p_{i}^{D} \triangleq p_{i} \cap P\left(\begin{array}{l}
\left(v_{\min } \leq|v| \leq v_{\text {max }}\right) \cap\left(\left|v-v_{J}\right| / t_{s}\right) \leq a_{\max } \\
\cap\left(v v_{f}\right) \geq-\xi_{u}
\end{array}\right)
$$

where $v$ and $v$, are assumed to be uniformly distributed random variables between $-v_{\text {moz }}$ and $v_{\text {cmax }}$ belonging to the Doppler velocity of clutter at the current and the preceding sampling time, respectively. Since the first event, $p_{i}$, uses velocity estimation based on position only measurements and the rest use Doppler velocity measurements directly, these events can be assumed to be independent Recalling that the undesired measurements in the resolution celis are also independent of each other, $p_{1}^{D}$ can be written as:

$$
p_{i}^{D}=p_{i} S_{1} S_{2} S_{3}
$$

where

$$
\begin{aligned}
S_{1} & =P\left(v_{\min } \leq|v| \leq v_{\max }\right) \\
& =1-\left(v_{\operatorname{man}} / v_{\mathrm{c} \max }\right), v_{\min }<v_{\mathrm{cmax}}<v_{\max } \\
S_{2} & =P\left(\left(\left|v-v_{j}\right| / t_{s}\right) \leq a_{\max }\right) \\
& =\left(\frac{a_{\max } t_{s}}{v_{c \max }}\right)\left(1-\frac{a_{\max } t_{s}}{4 v_{c \max }}\right), a_{\max } t_{s}<2 v_{\mathrm{cmax}} \\
S_{3} & =P\left(v v_{J} \geq-\xi_{v}\right) \\
& =0.5-\frac{\xi_{v v}}{2 v_{c \max }^{2}}\left(1+\ln \left(-\frac{v_{c \max }^{2}}{\xi_{v}}\right)\right), \xi_{v}>0
\end{aligned}
$$

Therefore, the overall FTIP in case of position plus Doppler velocity measurements, $P_{F T I}^{D}$ is calculated for both TI methods as:

$$
P_{F 77}^{\nu}=\left(1-\exp \left(-P_{F A} \zeta p_{2}^{D}\right)\right)\left(1-\exp \left(-P_{F A} \zeta p_{3}^{D}\right)\right)^{2}(13)
$$

Results of Track Initiation

In this section performance evaluations of $\mathrm{T}$ ! approaches are observed and quantified through simulations. Firstly, simulations to estimate TTIP, $P_{T T I}$, will be considered. The system requirement about TTIP is $P_{T T I} \geq 0.70$ at $M=4$. The total number of Monte Carlo run is chosen as 1,000 to yield unbiased results. The transfer interval of all the extracted plots by the PAR in the search volume is chosen as $t_{\mathrm{s}}=2 \mathrm{~s}$. Maximum acceleration levels of these benchmark targets, $a_{\max }$ are 31,39 , $42,58,68$ and $70 \mathrm{~m} / \mathrm{s}^{2}$ respectively. The minimum velocity threshold value is calculated using the minimum acceleration level of the targets in manoeuvre, $a_{\min }$, as $v_{\min }=a_{\min } t_{\mathrm{o}}=20 \cdot 2=40 \mathrm{~m} / \mathrm{s}$ and the maximum velocity threshold is chosen as $v_{r a x}=1000 \mathrm{~m} / \mathrm{s}$. When the Doppler velocity measurement is used, the threshold $\xi_{v}$ is defined as 
$\xi_{\mathrm{v}} \triangleq\left(t_{a} a_{m, i n}\right)^{2}=(2 \times 20)^{2} \mathrm{~m}^{2} / \mathrm{s}^{2}$. The angular coverage of the PAR is selected as $\theta_{t_{4}}=-57^{\circ}$ b $\theta_{t_{4}}=53^{\circ}$ in azimuth; $\phi_{t_{1}}=0^{\circ}$ to $\phi_{t_{1}}=15^{\circ}$ in elevation. The $3 \mathrm{~dB}$ beamwidths for both angles is $0.5^{\circ}$ and the maximum unambiguous range is calculated as $125 \mathrm{~km}$. The radar used in this study has $\% 95$ probability of detection for the signal-to-noise ratio (SNR) obtained at the maximum unambiguous range. For this SNR value, the measurement accuracies of the PAR is calculated as $20 \mathrm{~m}$ in range and $0.54 \mathrm{mrad}$ in angles. After solving Doppler velocity ambiguity, measurement accuracy in Doppler velocity is calculated as $40 \mathrm{~m} / \mathrm{s}$ for the given SNR and the dwell time. For the logic-based TI method, radius parameter is chosen as $r_{0}=500 \mathrm{~m}$ to meet the minimum TTIP requirement.

According to these parameters, simulations are done to get TTIP performance for both TI methods with the extracted plots of the benchmark targets. The results are given in Table 1.

Table 1 TTIP values of the TI methods (RB: the rulebased TI, LB: the logic-based TI, PO: position only measurements, PD: position plus Doppler velocity measurements)

\begin{tabular}{|c|c|c|c|c|}
\hline $\begin{array}{c}\text { Target } \\
\text { No }\end{array}$ & RB,PO & LB,PO & RB,PD & LB,PD \\
\hline 1 & 0.86 & 0.86 & 0.77 & 0.80 \\
\hline 2 & 0.85 & 0.85 & 0.84 & 0.84 \\
\hline 3 & 0.85 & 0.85 & 0.83 & 0.84 \\
\hline 4 & 0.84 & 0.86 & 0.70 & 0.70 \\
\hline 5 & 0.80 & 0.85 & 0.76 & 0.82 \\
\hline 6 & 0.80 & 0.86 & 0.70 & 0.75 \\
\hline
\end{tabular}

The results show that both methods produce similar TTIP values. When the Doppler velocity reports are used for TI process, due to the additional tests, TTIP is reduced depending on the manoeuvre capability and Doppler profile of the benchmark targets. The average rate of reduction of TTIP is calculated as \%7.7. Even if the Doppler velocity measurement is used, system requirement $P_{T T \prime} \geq 0.70$ is satisfied.

For the evaluation of analytical FTIPs, various probability of false alarm values are selected as $1 \times 10^{6}, 5 \times 10^{-6}, 1 \times 10^{-5}, 5 \times 10^{-5}$ and $1 \times 10^{-4}$ (per resolution cell). The total volume of the search region is calculated using the radar parameters given above as $V=2.57 \times 10^{5} \mathrm{~km}^{3}$. The maximum Doppler velocity of clutter is practically chosen as $v_{\mathrm{cmax}}=15 \mathrm{~m} / \mathrm{s}$. To evaluate the FTIPs of the rule-based TI method, different $a_{\max }$ values are used.

Table 2 presents the FTIP values of the rule-based TI with position only measurements for different values of $a_{\max }$ and $P_{F_{f}}$.
Table 2 FTIPs of the rule-based TI with position only measurements

\begin{tabular}{|c|ccccc|}
\cline { 2 - 6 } \multicolumn{1}{c|}{} & \multicolumn{5}{|c|}{$P_{F \lambda}$} \\
\cline { 2 - 6 } \multicolumn{1}{c|}{} & $1 \mathrm{E}-6$ & $5 \mathrm{E}-6$ & $1 \mathrm{E}-5$ & $5 \mathrm{E}-5$ & $1 \mathrm{E}-4$ \\
\hline \hline$a_{\text {ruas }}$ & \multicolumn{5}{|c|}{$P_{F T I}$} \\
\hline 31 & $2.43 \mathrm{E}-14$ & $3.04 \mathrm{E}-12$ & $2.43 \mathrm{E}-11$ & $2.99 \mathrm{E}-09$ & $2.35 \mathrm{E}-08$ \\
\hline 58 & $7.42 \mathrm{E}-14$ & $9.26 \mathrm{E}-12$ & $7.39 \mathrm{E}-11$ & $9.10 \mathrm{E}-09$ & $7.15 \mathrm{E}-08$ \\
\hline 70 & $1.01 \mathrm{E}-13$ & $1.27 \mathrm{E}-11$ & $1.01 \mathrm{E}-10$ & $1.25 \mathrm{E}-08$ & $9.78 \mathrm{E}-08$ \\
\hline
\end{tabular}

When the Doppler velocity measurement is used for TI, the obtained FTIPs are given in Table 3

Table 3 FTIPs of the rule-based TI with position and Doppler velocity measurements

\begin{tabular}{|c|ccccc|}
\cline { 2 - 6 } \multicolumn{1}{c|}{} & \multicolumn{5}{c|}{$P_{F d}$} \\
\cline { 2 - 6 } \multicolumn{1}{c|}{} & $1 \mathrm{E}-6$ & $5 \mathrm{E}-6$ & $1 \mathrm{E}-5$ & $5 \mathrm{E}-5$ & $1 \mathrm{E}-4$ \\
\hline$a_{\text {mas }}$ & \multicolumn{5}{|c|}{$P_{F T r}$} \\
\hline 31 & $1.22 \mathrm{E}-16$ & $1.52 \mathrm{E}-14$ & $1.22 \mathrm{E}-13$ & $1.52 \mathrm{E}-11$ & $1.21 \mathrm{E}-10$ \\
\hline 58 & $1.77 \mathrm{E}-15$ & $2.21 \mathrm{E}-13$ & $1.77 \mathrm{E}-12$ & $2.20 \mathrm{E}-10$ & $1.75 \mathrm{E}-09$ \\
\hline 70 & $3.66 \mathrm{E}-15$ & $4.57 \mathrm{E}-13$ & $3.65 \mathrm{E}-12$ & $4.55 \mathrm{E}-10$ & $3.61 \mathrm{E}-09$ \\
\hline
\end{tabular}

Similarly, the FTIPs belonging to the logic-based TI method with position only measurements and position plus Doppler velocity measurements are given in Table 4 and Table 5, respectively. In this method, due to the gate with fixed radius, FTIP is independent of $a_{\text {rpgr }}$.

Table 4 FTIPs of the logic-based TI with position only measurements

\begin{tabular}{|c|c|c|c|c|}
\hline $1 \mathrm{E}-6$ & $5 \mathrm{E}-6$ & $1 \mathrm{E}-5$ & $5 \mathrm{E}-5$ & $1 \mathrm{E}-4$ \\
\hline \multicolumn{5}{|c|}{$P_{\mu, 7}$} \\
\hline $8.93 \mathrm{E}-14$ & $.11 \mathrm{E}-11$ & $94 \mathrm{E}-11$ & $.10 \mathrm{E}-08$ & $.61 \mathrm{E}-08$ \\
\hline
\end{tabular}

Table 5 FTIPs of the rule-based TI with position and Doppler velocity measurements

\begin{tabular}{|c|c|c|c|c|c|}
\hline & \multicolumn{5}{|c|}{$P_{F A}$} \\
\hline & $1 \mathrm{E}-6$ & $5 \mathrm{E}-6$ & $1 \mathrm{E}-5$ & $5 \mathrm{E}-5$ & $1 \mathrm{E}-4$ \\
\hline$a_{\text {mas }}$ & & & $P_{F T I}$ & & \\
\hline 31 & $4.47 \mathrm{E}-16$ & $5.59 \mathrm{E}-14$ & $4.47 \mathrm{E}-13$ & $5.57 \mathrm{E}-11$ & $4.44 \mathrm{E}-10$ \\
\hline 58 & $2.13 \mathrm{E}-15$ & $2.66 \mathrm{E}-13$ & $2.13 \mathrm{E}-12$ & $2.65 \mathrm{E}-10$ & $2.11 \mathrm{E}-9$ \\
\hline 70 & 3.22E-15 & $4.02 \mathrm{E}-13$ & $3.21 \mathrm{E}-12$ & $4.00 \mathrm{E}-10$ & $3.18 \mathrm{E}-09$ \\
\hline
\end{tabular}

Since the methodology to incorporate Doppler velocity is the same for both $\mathrm{TI}$ methods, the obtained rates of reduction of FTIPs are approximately 
between 27 and 194 depending on the values of $a_{\text {mar }}$ while provoding a TTIP which is obtained as $P_{T I} \geq 0.70$ given in Table 1 . Increasing the minimum velocity threshold towards the assumed maximum Doppler velocity of clutter provides less FTIPS, obviously. However, since TTIPs are also reduced so that it cannot meet the minimum requirement, there exists a trade-off.

\section{Track Maintenance (TM)}

Since PARs are capable of using an adaptive sampling policy through agile beam positioning suitable control of a PAR has the potential fo significantly improving the track maintenance performance. The problem includes the proper control of beam positioning of a PAR using Doppler velocity measurements along with the three dimensional position measurements. In doing so, selection of next sampling time is of crucial importance. Especially for multiple targets and cluttered environments, increasing the duration between two sampling time i.e. track update interval, denotes less signal processing computations and lower radar energy enabling simultaneous tracking of more targets However, increasing the interval between successive sampling time also leads in increments of the state estimation errors. Therefore, bearing in mind such a tradeoff, the main purpose should be increasing track update interval while keeping the levels of other performance criteria satisfactory. For this reason. Interacting Multiple Model Probabilistic Data Association Filter (IMMPDAF) algorithm satisfies the requirements above. In this algorithm, three dimensional position measurements are usually used [6]. However, the major discriminant of clutter from the desired target with relatively higher velocities is the Doppler velocity measurement [4]. In the open literature, there is no study in which the Dopple measurements extracted by a PAR are included in IMMPDAF with adaptive sampling to track highly manoeuvring targets in complex environments. The aim of this section is to show the further improvement on the performance of the developed IMMPDAF algorithm with three modes if Doppler velocity measurements along with three dimensional position measurements are used. The performance criteria of the algorithm are: (i) average true target detection percantage; (ii) average track lost percantage; (iii) average position estimation error; (iv) average track update interval.

Track Maintenance Algorithm

IMMPDAF algorithm consists of two main stages: measurement association, i.e. PDA unit and track update (filtering), i.e. IMM unit $[5,6]$. The evolution of the target for both is modeled in cartesian coordinates with more than one models which correspond to the different types of target motions [5,6]. Here, IMM with three models is considered. The first model is a nearly constant velocity motion with low level of process noise. The second one has a relatively higher level of process noise corresponding to an on-going manoeuvre. Finally, the third mode of operation with a Wiener process acceleration model corresponds to the target starting/ending a manoeuvre. The transitions between the modes are selected as a class of a semi-Markov process which produces timevarying model transition probabilities. The covariances of the process noise levels used in IMM are chosen based on the levels of maximum acceleration and maximum acceleration increment per unit time (jerk) corresponding to the relevant model. To overcome the degrading effects of clutter, PDA method with the appropriate parameters is adopted. The measurements are range, azimuth, elevation and Doppler velocity. Since the given benchmark [7] does not supply Doppler information, a similar benchmark environment which also produces Doppler velocity measurements is set up. Conversion of the measurements from spherical coordinates to cartesian coordinates is not used in this study. Hence, the measurement equation is a nonlinear function of the state vector. Therefore, an extended Kalman filter based PDA method is implemented. To select next sampling time, an existing and most popular method is used [5]. According to this method, track update interval is chosen from a predetermined set as the largest one such that the predicted standard deviations of the angle innovations are smaller than a certain threshold value related to a fraction of the beamwidth of the PAR. The next beam is pointed to the predicted azimuth and elevation angles at the next sampling time chosen by the algorithm.

\section{Simulations and Results of TM}

The performance comparison of an IMMPDAF tracker with $P O$ and $P D$ cases are investigated here. The state vector has nine dimensions having position, velocity and acceleration estimates in $3 D$. Since the measurement vector includes Doppler velocity, nonlinearity occurs at the measurement equation. Therefore an extended Kalman filter based PDA structure is employed. For this reason, at each update, the measurement equation is linearized using the evaluation of the Jacobians around the predicted state vector. As to the IMM models, process noise standard deviation for the nearly constant velocity model is chosen as $\sigma_{\mathrm{v}}=2 \mathrm{~m} / \mathrm{s}^{2}$. For on-going manoeuvre model, it is selected as $\sigma_{v_{2}}=30 \mathrm{~m} / \mathrm{s}^{2}$. Finally, standartd deviation value for the Wiener process acceleration model is calculated as $\sigma_{n_{3}}=\min \left(0.5 \dot{a}_{\max } \delta_{k}, a_{\max }\right) \quad$ where $a_{\max }$ herein is selected as $70 \mathrm{~m} / \mathrm{s}^{2}, \delta_{k}$ is the calculated next sampling interval at the $k^{\text {th }}$ sampling time and $\dot{a}_{\text {max }}$ is the jerk term as $\dot{a}_{m a x}=60 \mathrm{~m} / \mathrm{s}^{3}$ [6]. The initial mode probabilities are selected as $\left.\mid \begin{array}{lll}0.5 & 0.25 & 0.25\end{array}\right]^{T}$. The mode probabilitiy matrix, $\Pi\left(\delta_{k}\right)$, is updated according to:

$$
\Pi\left(\delta_{k}\right)=\left|\begin{array}{ccc}
1-0.05 \delta_{k} & 0.05\left(1-p_{11}\left(\delta_{k}\right)\right) & \left.0.95-0.95 p_{11}\left(\delta_{k}\right)\right) \\
0.05\left(1-p_{22}\left(\delta_{k}\right)\right) & 1-0.25 \delta_{k} & 0.95\left(1-p_{22}\left(\delta_{k}\right)\right) \\
0.33\left(1-p_{33}\left(\delta_{k}\right)\right) & 0.67\left(1-p_{3}\left(\delta_{k}\right)\right) & \max \left(1-\delta_{k}, \delta_{k}\right)
\end{array}\right|
$$


where $p_{i t}$ is the probability of transition from $i^{\text {th }}$ mode to the same mode, $\delta_{k}^{\min }$ is the minimum track update interval selected as $\delta_{k}^{\text {min }}=0.1 \mathrm{~s}$. Since the motion parameters of the target vary due to manoeuvres, an adaptive sampling policy is required. The next sampling interval, $\delta_{k}$, is selected from preset values given as $\mathbf{t}=\left[\begin{array}{llllll}3 & 2.5 & 2 & 1 & 0.5 & 0.1\end{array}\right]^{T} . \quad$ The measurement noise is AWGN with the standard deviations of the measurement accuracies given in the preceding section. The standard deviation of clutter based Doppler velocity measurements is realistically selected as $50 \mathrm{~m} / \mathrm{s}$. Probability of false alarm, $P_{F A}$, is set to $1 \times 10^{-4}$ (per resolution cell). The range gate size for tracking is taken as $1.5 \mathrm{~km}$. Since the validation gate threshold, $\gamma$, used in PDAF depends on the dimension of measurement vector and the validation gate probability, $P_{G}$, for $P_{G}=0.99$,

$\gamma$ values are found using Chi-Square table as 11.3 for position onfy measurements and $\mathbf{1 3 . 3}$ for position plus Doppler velocity measurements case. The angle thresholds used in the selection of $\delta_{k}$ is taken as ten times the angular accuracy. The simulation results based on 100 Monte Carlo run is given below.

Table 6 The results about the performance criteria for $\mathrm{PO}$ and PD measurements

\begin{tabular}{|c|c|c|c|c|c|c|c|}
\hline \multirow{2}{*}{\multicolumn{2}{|c|}{$\begin{array}{c}\text { Performance } \\
\text { Criteria }\end{array}$}} & \multicolumn{6}{|c|}{ Target No } \\
\hline & & 1 & 2 & 3 & 4 & 5 & 6 \\
\hline \multirow{2}{*}{$\begin{array}{l}\text { Track update } \\
\text { Interval (s) }\end{array}$} & PO & 2.41 & 2.22 & 2.23 & 2.06 & 2.27 & 2.07 \\
\hline & PD & 2.46 & 2.30 & 2.37 & 2.14 & 2.37 & 2.18 \\
\hline \multirow{2}{*}{$\begin{array}{l}\text { Track Lost } \\
\text { Percantage }\end{array}$} & $\mathrm{PO}$ & 7 & 8 & 9 & 10 & 10 & 10 \\
\hline & PD & 1 & 5 & 6 & 10 & 9 & 9 \\
\hline \multirow{2}{*}{$\begin{array}{l}\text { Position } \\
\text { Error (m) }\end{array}$} & PO & 51 & 46 & 55 & 35 & 64 & 56 \\
\hline & PD & 53 & 45 & 52 & 40 & 60 & 60 \\
\hline \multirow{2}{*}{\begin{tabular}{|l} 
True Track \\
Detection \\
Percantage \\
\end{tabular}} & $\mathrm{PO}$ & 94 & 94 & 95 & 95 & 95 & 95 \\
\hline & PD & 94 & 95 & 95 & 95 & 95 & 95 \\
\hline
\end{tabular}

In Table 6, with the incorporation of Doppler velocity measurement, the average track update interval is increased by the rate of between $1.86 \%$ and $6.08 \%$ resulting in an acceptable increment of RMS position error by the average rate of $1.78 \%$. Since the incorporation of Doppler velocity measurements increases the track update interval as shown in Table 6, the number of dwells needed is also reduced causing energy saving by the rate of upto $6.08 \%$.

\section{Conclusions}

In this paper, we presented the effects of incorporating Doppler velocity measurement extracted by a phased array radar into track initiation and maintenance algorithms. Firstly, a realistic evaluation technique that avoids time-consuming simulations for track initiation has been demonstrated. This analytical expressions can be used to select the signal processing parameters that meet the track initiation requirements such as false track initiation probability and true track initiation probability. It indicates that using Doppler velocity reports along with 3-D position measurements in complex environments leads to significant decrease on false track initiation probability with an acceptable true track initiation probability. Besides, the effects of including Doppler velocity measurements into IMMPDAF estimator with adaptive sampling is investigated and the performance improvement is compared with those cases where only three dimensional position measurements are used. It is been observed that using Doppler velocity measurements in track maintenance leads to increased track update intervals resulting in energy conservation.

\section{References}

[1] Shalom, Y.B., Chang, K.C., Shertukde, H.M., Performance Evaluation of Cascaded Logic for Track Formation in Clutter, IEEE Trans. On Aerospace and Electronic Systems, 25(6), 1989, pp.873-877.

[2] Shalom, Y.B., Li, X., Effectiveness of the Likelihood Function in Logic Based Track Formation, IEEE Trans. On Aerospace and Electronic Systems, 27(1), 1991, pp.184-187.

[3] Hu, Z., Leung, H., Blanchette, M., Statistica Performance Analysis of Track Initiation Techniques, IEEE Trans. On Signal Processing, 45(2), 1997, pp.445-456.

[4] Farina, A., Pardini, S., Track-While Scan Algorithm in a Clutter Environment, IEEE Trans. On Aerospace and Electronic Systems, 14, 1978, pp.769778 .

[5] Kirubarajan,T., Shalom, Y.B., Daeupour, E., Adaptive Beam Pointing Control of a Phased Array Radar in the Presence of ECM and False Alarms Using IMMPDAF, Proceedings of American Control Conference, Seattle, WA, 1995, pp.2616-2620.

[6] Shalom, Y.B., Li, Xiao-Rong, MultitargetMultisensor Tracking: Principles and Techniques, 2. Edition, Danver, 1995.

[7] Blair, W.D., Watson, G.A., Hoffman, S.A., Benchmark Problem for Beam Pointing Control Phased Array Radar Against Maneuvering Targets: Proceedings of American Control Conference. TP 5 4:00, 1994, pp.2071-2075 